\title{
Development of a Hierarchy-Integrated Simulation Code for Toroidal Helical Plasmas, TASK3D
}

\author{
Masahiko SATO, Shinichiro TODA, Yuji NAKAMURA ${ }^{1)}$, Kiyomasa WATANABE, \\ Atsushi FUKUYAMA ${ }^{2)}$, Sadayoshi MURAKAMI ${ }^{2)}$, Masayuki YOKOYAMA, Hisamichi FUNABA, \\ Hiroshi YAMADA and Noriyoshi NAKAJIMA \\ National Institute for Fusion Science, Toki 509-5292, Japan \\ ${ }^{1)}$ Graduate School of Energy Science, Kyoto University, Kyoto 611-0011, Japan \\ ${ }^{2)}$ Graduate School of Engineering, Kyoto 606-8501, Japan
}

(Received 21 November 2007 / Accepted 18 March 2008)

\begin{abstract}
The present status of the development of a hierarchy-integrated simulation code for toroidal helical plasmas, TASK3D, is reported. TASK3D is developed by extending the integrated modeling code for tokamak plasmas, Transport Analyzing System for tokamaK (TASK) [A. Fukuyama et al., Proc. of 20th IAEA Fusion Energy Conf. (Villamoura, Portugal, 2004) IAEA-CSP-25/CD/TH/P2-3]. In order to extend TASK to be applicable for threedimensional configurations, a new module for the radial electric field in general toroidal configurations has been developed and implemented. As a first test for this implementation, numerical simulations for the time evolution of temperature and electric field are conducted on the basis of an LHD experimental result, by a successful combination of a diffusive transport module and the implemented electric field module.
\end{abstract}

(c) 2008 The Japan Society of Plasma Science and Nuclear Fusion Research

Keywords: integrated simulation, helical plasma, radial electric field

DOI: $10.1585 /$ pfr.3.S1063

\section{Introduction}

In order to systematically clarify confinement physics in toroidal magnetic confinement systems, a hierarchyrenormalized simulation concept is being developed under domestic and international collaborations with universities and institutes [2]. The hierarchy-renormalized simulation model in toroidal magnetic confinement systems consists of a hierarchy-integrated and a hierarchy-extended simulation approach. The former approach is based mainly on a transport simulation combining various simplified models that describe physical processes in different hierarchies. This is suitable for investigating complete temporal behavior of experimentally observed macroscopic physical quantities. The latter approach includes fluid core plasma description [3-6], kinetic core plasma description [7], and peripheral fluid/kinetic description [8,9]. These descriptions focus on the mutual interaction among neighboring hierarchies in a rigorous way. For the hierarchy-integrated simulation approach, an integrated modeling code for threedimensional configurations (TASK3D) is being developed on the basis of an integrated modeling code for tokamak plasmas, Transport Analyzing System for tokamaK (TASK) [1], which was developed in Kyoto University. In order to extend the TASK code to be applicable for threedimensional configurations, the transport equations for rotational transform [2] and radial electric field $\left(E_{r}\right)$ have been reformulated by considering the three-dimensional

author'se-mail: masahiko@nifs.ac.jp nature of configurations. With this new formulation, a new module for $E_{r}$ (ER module) has been developed and implemented. In this paper, the present status of the first-stage of the development of TASK3D - implementation of the ER module in TASK3D and evaluation of its applicability - is reported, by performing test simulations based on an LHD experimental result.

This paper is organized as follows. In Section 2, the module structure of TASK3D is briefly described, and the governing equation for $E_{r}$ in three-dimensional configurations is explained as the core feature of the ER module. In Section 3, we show the results of test simulations obtained by successful combinating a one-dimensional (radial) diffusive transport module, TR module, (originally used in TASK), and the newly implemented ER module, where the $E_{r}$ profile is determined using the ambipolar condition. Finally, Section 4 is devoted to a summary and future plans of TASK3D development.

\section{Numerical Model in TASK3D}

TASK is an integrated modeling code for tokamak plasmas, which was developed in Kyoto University [1]. The TASK has a modular structure, allowing us to conduct simulations using an individual module or combination of some modules according to the user's objective. For the extension of TASK to TASK3D for simulating three-dimensional configurations, implementation of new modules (reflecting three-dimensionality of config- 


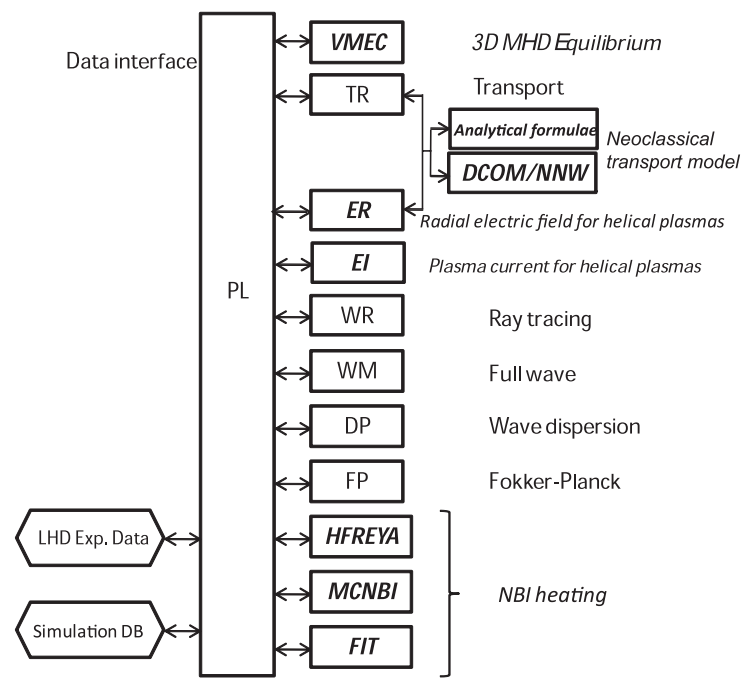

Fig. 1 Module structure of TASK/3D. Modules denoted by italics have been developed/implemented.

urations) into the TASK has been progressed as shown in Fig. 1 (as indicated by italics). At the current stage of the development of TASK3D, the extension of the TR module is the primary objective. In the TR module, the following particle and heat transport equations are solved;

$$
\begin{array}{r}
\frac{\partial}{\partial t}\left(n_{s} V^{\prime}\right)=-\frac{\partial}{\partial r}\left(V^{\prime}\langle|\nabla r|\rangle n_{s} V_{s}\right. \\
\left.-V^{\prime}\left\langle|\nabla r|^{2}\right\rangle D_{s} \frac{\partial n_{s}}{\partial r}\right)+S_{s}, \\
\frac{1}{V^{\prime 5 / 3}} \frac{\partial}{\partial t}\left(\frac{3}{2} n_{s} T_{s} V^{\prime 5 / 3}\right) \\
=-\frac{1}{V^{\prime}} \frac{\partial}{\partial r}\left(V^{\prime}\langle|\nabla r|\rangle \frac{3}{2} n_{s} T_{s} V_{E s}\right. \\
\left.-V^{\prime}\left\langle|\nabla r|^{2}\right\rangle n_{s} \chi_{s} \frac{\partial T_{s}}{\partial r}\right)+P_{s},
\end{array}
$$

where $n_{s}$ is the density, $T_{s}$ is the temperature, and $s$ expresses the particle species. $V_{E s}=V_{K s}+(3 / 2) V_{s}$, where $V_{K s}$ is the heat pinch velocity and $D_{s}, \chi_{s}$, and $V_{s}$ are the particle diffusion coefficient, thermal diffusion coefficient, and particle pinch velocity, respectively, which consist of a neoclassical part and an anomalous part. $S_{s}$ is the particle source and $P_{s}$ is the energy source (or sink). $t, r, V$ are the time, minor radius variable of magnetic surface, and volume enclosed by the magnetic surface, respectively. The prime denotes the derivative with respect to $r$, and \langle\rangle represents the magnetic surface average. The original TR module in TASK also solves the equation of the magnetic field for analyzing the plasma current. However, the equation of the magnetic field is derived by assuming the axisymmetry of configurations; therefore it cannot be applied for analyzing the plasma current in helical plasmas. Hence, the equation for the rotational transform in general toroidal configurations has been reformulated and a new module (EI module) was developed and tested [2].
Another important extension of the TR module for helical plasmas is to consider the effect of $E_{r}$ since the ambipolar condition is not satisfied intrinsically due to the non-axisymmetry of helical plasmas, and $E_{r}$ is determined by neoclassical transport. Hence, the following equation for $E_{r}$ should be employed for solving the temporal evolution of $E_{r}$,

$$
\begin{aligned}
\epsilon_{0} \epsilon_{r}\left\langle|\nabla r|^{2}\right\rangle \frac{\partial}{\partial t} \frac{\partial \Phi_{0}}{\partial r}= & \sum_{s}\left\langle\boldsymbol{B}_{x} \cdot \nabla \cdot \Pi_{s}\right\rangle \\
& -\sum_{s}\left\langle\boldsymbol{B}_{x} \cdot \boldsymbol{M}_{s}\right\rangle \\
& +e_{f}\left\langle n_{f} \boldsymbol{u}_{f} \cdot \nabla r\right\rangle,
\end{aligned}
$$

where $\Phi_{0}$ is the electrostatic potential, $\Pi_{s}$ is the viscous tensor, and $\boldsymbol{M}_{s}$ is the momentum source. The third terms on the right hand side is the fast particle term resulting from neutral beam injection (NBI), where $e_{f}, n_{f}$ and $\boldsymbol{u}_{f}$ are the charge, density, and velocity for the fast particles, respectively. In Eq. (3), $\boldsymbol{B}_{x}$ is defined by

$$
\boldsymbol{B}_{x}=\frac{\nabla r \times \boldsymbol{B}}{B^{2}}+\left(\frac{\mathrm{d} \phi_{T}}{\mathrm{~d} r}\right)^{-1}\left(\frac{g_{2}}{B^{2}}-\frac{\left\langle g_{2}\right\rangle}{\left\langle B^{2}\right\rangle}\right),
$$

where $\phi_{T}$ is the toroidal magnetic flux, $\boldsymbol{B}$ is the magnetic field, and $B=|\boldsymbol{B}|$. The relative permittivity $\epsilon_{r}$ is given by

$$
\epsilon_{r}=1+\frac{c^{2}}{\left\langle\left|\nabla \phi_{T}\right|^{2}\right\rangle}\left\{\left\langle\frac{\left|\nabla \phi_{T}\right|^{2}}{v_{\mathrm{A}}^{2}}\right\rangle+\left\langle\frac{\left(g_{2}\right)^{2}}{v_{\mathrm{A}}^{2}}\right\rangle-\frac{\left\langle g_{2}\right\rangle^{2}}{\left\langle v_{\mathrm{A}}^{2}\right\rangle}\right\},
$$

where $v_{\mathrm{A}}$ is the Alfvén velocity. In Eq. (4), $g_{2}$, which describes Pfirsch-Schluter current, is determined from

$$
\boldsymbol{B} \cdot \nabla\left(\frac{g_{2}}{B^{2}}\right)=\boldsymbol{B} \times \nabla \phi_{T} \cdot \nabla\left(\frac{1}{B^{2}}\right) .
$$

The time scale of the time evolution of $E_{r}$ is much shorted than that for the density and temperature. Therefore, the time step in the calculation of $E_{r}$ has to be smaller than that for the density and temperature. At the current stage of development of TASK3D, the ER module has been developed and implemented, where $E_{r}$ is determined from the ambipolar condition by assuming the stationary state of Eq. (3) in order to avoid the problem of different time scales, and neglecting the source and fast particle terms in Eq. (3). The extension of the ER module from the ambipolar condition to the equation of temporal evolution, Eq. (3), will be performed in the future (cf., Sec. 4).

Estimation of neoclassical particle fluxes is required for obtaining the ambipolar $E_{r}$ in the ER module. For this purpose, two approaches have been considered for implementation in TASK3D. One is to employ simple analytic formulae [5], and the other is to utilize the database of neoclassical diffusion coefficients being constructed using Diffusion Coefficient Calculator by Monte Carlo Method/Neural NetWork (DCOM/NNW) [10], based on the Monte Carlo code, DCOM. Even at this stage, TASK3D simulations for more real situations (e.g., to complete a "full time" simulation of an experimental discharge) 


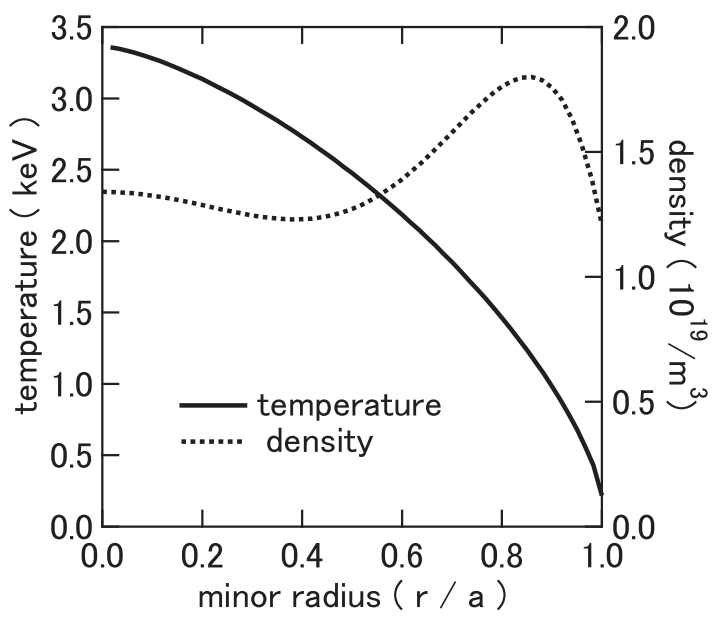

Fig. 2 Initial profiles of temperature and density. The ion temperature and density are the same as those of electrons.

of course, require many iterations. In such a case, relatively quick calculations in each module are desirable, while keeping the important physical aspects. In this sense, both approaches have certain advantages and disadvatages. For example, the analytic formulae in Ref. [11] assume a model magnetic field with a single helicity, which strongly limits its rigorous applicability for wide range equilibria in LHD. However, by employing such analytical formulae, it is shown that some physical phenomena are properly simulated, such as in the case for providing the neoclassical transport level in anomalous transport modeling [12], and for explaining the bifurcation nature of $E_{r}$ that was experimentally observed/verified in LHD [13]. Investigations are conducted to obtain more accurate analytical formulae for neoclassical transport applicable to wide range equilibria [14], and it is useful to incorporate such studies in TASK3D. On the other hand, construction of the database of neoclassical diffusion coefficients using DCOM/NNW will take a long time to be fully applicable to wide range parameters realized/expected in LHD experiments, even though DCOM can include the detailed magnetic configuration properties. Currently, these two options for calculating the neoclassical fluxes have been implemented in the ER module, which is schematically shown in Fig. 1. In between these two options, the usage of the General Solution of the Riple Averaged Kinetic Equation (GSRAKE) code [15] for constructing the neoclassical database should be considered, since it can treat multiple helicity effects (but not as detailed as in DCOM) with less computation time.

\section{Test Simulation by Combining of TR and ER Modules}

In order to check the applicability of the ER module, test simulations have been performed by using the combination of the TR (originally in TASK) and newly implemented ER module. In this section, some example results

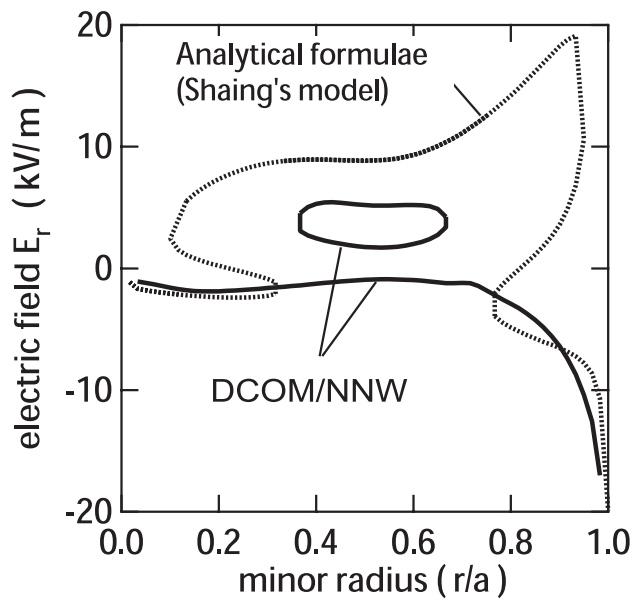

Fig. 3 Comparison of $E_{r}$ profiles between cases based on the analytical formulae and DCOM/NNW, where $E_{r}$ is calculated from the ambipolar condition using the initial profile of the density and temperature as shown in Fig. 2.

of such test simulations are shown. TASK3D has been extended to read LHD experimental data in Ufile format. For the simulation results shown in this section, LHD experiment data (shown in Fig. 2, where $a$ is the plasma minor radius) is used for initial profiles of the simulation. The characteristic parameters of this example discharge are $R_{\mathrm{ax}}=3.60 \mathrm{~m}, B=2.8 \mathrm{~T}$, and the maximum volume average beta value is about $0.65 \%$, where $R_{\mathrm{ax}}$ denotes the position of the vacuum magnetic axis. The ion temperature $\left(T_{\mathrm{i}}\right)$ is assumed to be the same as the electron temperature $\left(T_{\mathrm{e}}\right)$ at the initial state. $T_{\mathrm{e}}, T_{\mathrm{i}}$, and $E_{r}$ are calculated using the combination of the TR and ER modules. Other variables such as the density and rotational transform are fixed. The anomalous transport coefficient is a priori assumed to be $\chi^{\text {an }}=0.6 \mathrm{~m}^{2} / \mathrm{s}$.

Figure 3 shows an $E_{r}$ profile calculated using the ambipolar condition of neoclassical particle fluxes based on the analytic formulae and DCOM/NNW, where the radial profiles of the density and temperature shown in Fig. 2 are used. Multiple roots are obtained using each approach. The radial range for the existence of multiple roots is wider for the approach based on analytic formulae. However, since the appearance of the electron root (positive $E_{r}$ ) is sensitive to a small change in plasma parameters (reflecting the nonlinear dependence of neoclassical particle fluxes on $E_{r}$ ), it can be considered that both approaches provide a reasonable agreement. The amplitude of the predicted negative $E_{r}$ (ion root) is fairly comparable between the two approaches.

Figure 4 shows the radial profiles of $T_{\mathrm{e}}, T_{\mathrm{i}}$, and $E_{r}$ at a stationary state, obtained based on analytical formulae (Fig. 4(a)) and DCOM/NNW (Fig.4(b)) for neoclassical transport calculations. There figures also show a fairly reasonable agreement between both approaches. This agreement of ambipolar $E_{r}$ between the two approaches might be attributed to the selection of a low-beta plasma in the configuration having a predominant helicity. These test 

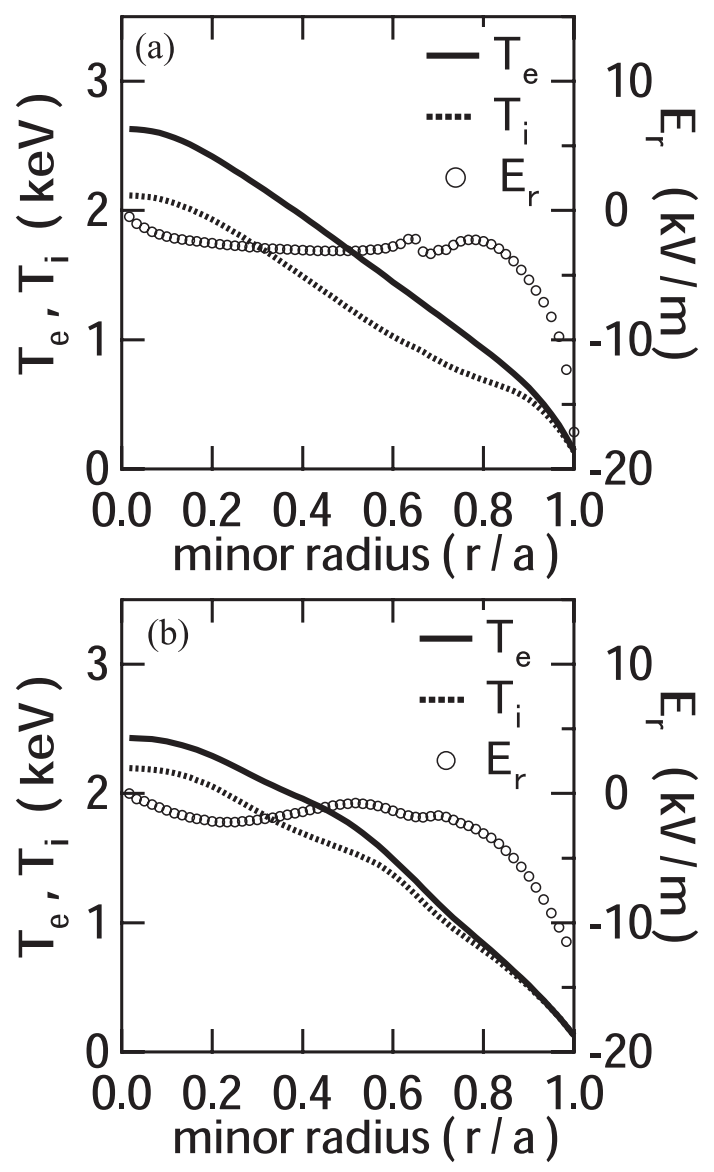

Fig. 4 Radial profiles of $T_{\mathrm{e}}, T_{\mathrm{i}}$, and $E_{r}$ at a stationary state for cases based on (a) analytical formulae and (b) DCOM/NNW for neoclassical transport calculations.

simulations based on the two approaches for neoclassical transport calculations have revealed the applicability and successful linkage of the TR and ER modules for an LHD experimental data. Validations of neoclassical transport models (valid range, accuracy versus computational time, etc.) require further accumulation of these test simulations for a quite large variety of parameter range achieved/expected in LHD experiments.

\section{Summary and Future Plans}

A hierarchy-integrated simulation code for toroidal helical plasmas, TASK3D, is being developed based on TASK, which is applicable for two-dimensional configurations. At the current stage of development of TASK3D, the ER module has been added to TASK3D, where $E_{r}$ is determined using the ambipolar condition. This is the important step of development, which enables the $E_{r}$ bifurcation nature of helical plasmas to be treated in the simulation. Two options for neoclassical flux calculations have been implemented, one is based on the analytical formulae and the other on a neoclassical diffusion coefficient database constructed using DCOM/NNW. In order to evaluate the applicability of the ER module, we conducted numerical simulations by establishing the linkage of LHD experiment data, which reveal the applicability and successful linkage of the TR module and ER modules. Extensive physics validation study will be performed on the basis of this implemented ER module for LHD experiments.

Calculations on the temporal evolution of the rotational transform and non-inductive current for an LHD experiment have already been performed $[2,16,17]$. In such simulations, the EI module was used together with the VMEC/BSC (MHD equilibrium consistent with the plasma current) module (cf., Fig. 1). In the next step of development of TASK3D, we will implement the EI module to enable fully inter-linked time evolutionary simulations for the rotational transform/plasma current. By performing this implementation, we will be able to simultaneously calculate the time evolution of temperature, density, $E_{r}$, and rotational transform. In addition, the currently used stationary ambipolar condition for determining $E_{r}$ will be replaced by the equation of time evolution of $E_{r}$, Eq. (3). Since the time scale of the time evolution of $E_{r}$ is much shorter than for other transport-related variables such as density and temperature, an implicit numerical method is necessary to solve the transport equations rapidly by avoiding the restrictions due to the Courant-Friedrichs-Lewy condition. The Newton method is considered to be a useful tool for solving implicitly nonlinear equations such as the transport equations. The implementation of the Newton solver in TASK3D is underway. After the addition of stable and fast Newton solver is completed, impurity module and heating modules will also be added to TASK3D for further development of the hierarchy-integrated simulation code for toroidal helical plasmas.

[1] A. Fukuyama et al., Proc. of 20th IAEA Fusion Energy Conf. (Villamoura, Portugal, 2004) IAEA-CSP-25/CD/TH/P2-3.

[2] Y. Nakamura et al., Fusion Sci. Technol. 50, 457 (2006).

[3] Y. Todo, Phys. Plasmas 13, 082503 (2006).

[4] A. Ishizawa and N. Nakajima, Phys. Plasmas 14, 040702 (2007).

[5] H. Miura, N. Nakajima, T. Hayashi et al., J. Plasma Phys. 72, 1095 (2006).

[6] R. Ishizaki, P. Parks, N. Nakajima et al., Phys. Plasmas 11, 4064 (2004).

[7] T.-H.Watanabe, H. Sugama and S. Ferrando-Margalet, Nucl. Fusion 47, 1383 (2007).

[8] Y. Tomita, R. Smirnov, T. Takizuka et al., J. Plasma Phys. 72, 1015 (2006).

[9] M. Kobayashi, N. Ohyabu, T. Muto et al., Fusion Sci. Tech. 52, 566 (2007).

[10] A. Wakasa et al., Jpn. J. Appl. Phys. 46, 1157 (2007).

[11] K.C. Shaing, Phys. Fluids 27, 1567 (1984).

[12] S. Toda et al., Nucl. Fusion 47, 914 (2007).

[13] K. Ida et al., Phys. Rev. Lett. 86, 5297 (2001).

[14] S. Nishimura, H. Sugama and Y. Nakamura, Fusion Sci. Tech. 51, 61 (2007).

[15] C.D. Beidler et al., Plasma Phys. Control. Fusion 37, 463 (1995).

[16] Y. Nakamura et al., Proc. of 21th IAEA Fusion Energy Conf. (Chengdu, China, 2006) IAEA-CN-149/TH/P7-1.

[17] Y. Nakamura et al., submitted to Plasma Fusion Res. 